Mots. Les langages du politique

Discours d'Amérique latine. Identités et conflits

\title{
Identités et conflits dans le discours politique latino-américain
}

Identity and conflicts in the Latin American political discourse

Identidades y conflictos en el discurso político latinoamericano

Églantine Samouth et Yeny Serrano

\section{OpenEdition}

Journals

Édition électronique

URL : https://journals.openedition.org/mots/22076

DOI : $10.4000 /$ mots. 22076

ISSN : 1960-6001

Éditeur

ENS Éditions

Édition imprimée

Date de publication : 30 novembre 2015

Pagination : 5-19

ISBN : 978-2-84788-776-1

ISSN : 0243-6450

\section{Référence électronique}

Églantine Samouth et Yeny Serrano, «Identités et conflits dans le discours politique latino-américain », Mots. Les langages du politique [En ligne], 109 | 2015, mis en ligne le 30 novembre 2015, consulté le 22 avril 2022. URL : http://journals.openedition.org/mots/22076 ; DOI : https://doi.org/10.4000/mots. 22076 


\section{Identités et conflits dans le discours politique latino-américain}

La question des identités collectives (socio-politiques, ethniques, linguistiques) semble se poser en Amérique latine de manière singulière. En effet, de par ses particularités historiques (colonisation par les Européens, indépendances, puis construction des États-Nations menées principalement par les élites blanches), et en raison de sa diversité ethnique, sociale et politique, le continent offre un terrain dans lequel les réflexions et les revendications autour de l'identité de certains groupes peuvent entraîner des enjeux de représentation politique ou d'accès à certaines ressources. Ces réflexions et ces revendications prennent forme dans des discours, objet auquel ce dossier s'intéresse plus particulièrement. Au-delà, ces derniers jouent un rôle dans la construction même de l'identité. L'objectif est ainsi d'analyser la manière dont les identités collectives se construisent, se disputent et se négocient à travers les discours politiques et/ou médiatiques.

Pour commencer, nous reviendrons sur la notion même d'identité. En vogue depuis quelques décennies, cette notion se caractérise par sa polysémie, voire son ambiguïté. Nous proposerons ainsi des éléments de réflexion sur cette question en portant une attention particulière aux approches discursives, avant de nous pencher sur la question de l'identité dans le contexte particulier de l’Amérique latine, puis de présenter les articles du dossier.

\section{L’identité, une notion « en quête d’identité »}

Si la notion d'identité fait actuellement l'objet de nombreuses réflexions, notamment au sein de la communauté universitaire, on peut toutefois constater qu'il est difficile de la définir. En effet, comme le souligne Jean-Claude Kaufmann dans l'article «Identité» du Dictionnaire des sciences humaines, ce concept assez récent - il s'est répandu dans les sciences humaines et sociales

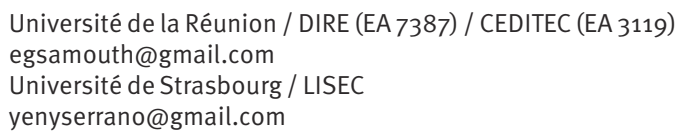


vers le milieu du $20^{\mathrm{e}}$ siècle - apparaît volontiers comme un présupposé, comme une évidence, que ce soit dans le langage courant ou dans le langage scientifique. Les efforts de définition dont il a fait l'objet se sont souvent révélés peu concluants, et jamais unanimes. Pour cet auteur, on peut néanmoins considérer que, a minima, la notion d'identité est majoritairement entendue dans les sciences humaines comme "l'interrogation, du point de vue de l'individu (ou du groupe), sur sa propre définition (“Qui suis-je?”) » (Kaufmann, 2006). Jean-François Dortier (2004) distingue trois principaux domaines d'étude dans la littérature actuelle sur l'identité : celui de l'identité collective - qui correspond à l'identité des nations, des minorités culturelles, religieuses ou ethniques -, celui de l'identité sociale - liée au rôle ou au statut dans la société, qui correspond à des facteurs comme le sexe, l'âge, la place dans la famille, la profession, etc. -, et celui de l'identité personnelle - liée à l'individu, où entrent en interaction les dimensions sociale, psychologique, consciente et inconsciente. C'est surtout le premier domaine, celui de l'identité collective ${ }^{1}$, qui nous intéresse dans le cadre de ce dossier.

On le voit, la notion d'identité recouvre un champ d'étude extrêmement vaste et intéresse de nombreuses disciplines (de la psychologie à la philosophie, en passant par la sociologie, l'anthropologie ou l'histoire). De plus, en traversant diverses disciplines et divers contextes historiques et socio-politiques, la notion s'est chargée de sens différents, ce qui n'est pas sans poser problème aux chercheurs qui s'y intéressent. On ne cherchera donc pas ici à retracer un historique du terme et des définitions qui en ont été données, ni à passer en revue les différentes approches de la notion, mais on s'attachera plutôt à rappeler quelques éléments de réflexion issus de travaux sur la question, afin de présenter la façon dont est conçue la notion d'identité dans le cadre de ce dossier.

La polyvalence du terme pose deux problèmes fondamentaux. Le premier tient au contexte d'utilisation. En effet, Rogers Brubaker affirme que c'est aux États-Unis, dans les années soixante, avec la naissance du mouvement des Black Panthers et d'autres mouvements ethniques, que le terme identité (identity) a été introduit dans l'analyse sociale. En tant que catégorie d'analyse, la notion semble donc avoir intéressé les chercheurs en sciences sociales une fois que des groupes sociaux ont commencé à se l'approprier en vue de revendications d'ordre politique et social. Par conséquent, comme le constate R. Brubaker, le terme identité constitue à la fois une "catégorie de pratique sociale» (utilisée par les acteurs sociaux ordinaires pour rendre compte d'eux-mêmes et par les politiques pour " persuader [...] certaines personnes qu'elles sont "identiques" entre elles en même temps que différentes d'autres personnes», Brubaker, 2001, p. 69) et une «catégorie d'analyse sociale et politique» (utilisée

1. Nous considérons que l'identité de genre fait partie des identités collectives, si on la traite du point de vue de la revendication de l’appartenance à un groupe dominé (voir infra). 
par les chercheurs en sciences humaines et sociales). En d'autres termes, alors que l'identité en tant que catégorie de pratique sociale désigne les revendications identitaires, l'identité en tant que catégorie d'analyse vise à décrire, comprendre et expliquer ces revendications dans une société donnée. Ce qui pose problème pour l'auteur, c'est l'utilisation du terme comme catégorie d'analyse qui ne se distingue quasiment pas de son utilisation comme catégorie de pratique sociale (ibid., p. 68-70). Cela a conduit certains chercheurs (Avanza, Laferté, 2005 ; Brubaker, 2001) à proposer de remplacer le terme identité par d'autres qui désignent les pratiques qu'ils entendent analyser lorsqu'ils parlent d'identité2, sans que cette proposition ait vraiment été suivie.

Le deuxième problème posé par la polyvalence du terme identité réside dans les cadres épistémologiques irréconciliables qui s'affrontent : soit l'identité est inhérente à l'individu, et c'est une sorte d'essence que l'on $a$; soit l'identité est une construction (collective ou individuelle) et dès lors on n'a pas une identité : on la construit en fonction du contexte et des relations sociales. On parle d'approches essentialistes dans le premier cas et d'approches constructivistes dans le second. Martina Avanza et Gilles Laferté (2005, p. 135) observent que les mouvements sociaux et politiques ont le plus souvent une conception essentialiste de l'identité : ils la perçoivent comme une donnée intangible, non négociable. C'est le cas par exemple de certains intellectuels latino-américains qui considèrent qu'il existe une identité latino-américaine authentique qu'il faut chercher à retrouver (voir infra).

Dans la conception constructiviste de l'identité qui, selon Eline Versluys, doit son émergence au «tournant linguistique» des sciences humaines et sociales, le langage et le discours jouent un rôle essentiel (2007, p. 92-93). En effet, au lieu de considérer que l'identité s'affiche ou est représentée à travers le langage, comme s'il y avait une identité préexistante, l'approche constructiviste postule que l’identité est construite, du moins en partie, au moyen du langage. Paul Kroskrity définit ainsi l’identité comme «la construction linguistique

2. Brubaker propose le terme d'identification, «dépourvu des connotations réifiantes du terme “identité" " - en ce qu'il désigne un processus complexe et non un état - et qui présente l'avantage d'inviter «à spécifier quels sont les agents qui procèdent à l'identification » $(2001$, p. 75$)$; le terme d'autocompréhension pour désigner la représentation à la fois cognitive et affective qu'on a de soi-même et du monde social, cette représentation pouvant être stable ou varier dans le temps et dans l'espace ; et celui de communalité pour désigner une forme spécifique d'autocompréhension relevant de la collectivité. Appartenir à un groupe signifie également que l'on se sent différent, voire que l'on nourrit une antipathie à l'égard des personnes extérieures (Brubaker, 2001, p.75-79). À la suite des travaux de Brubaker, M. Avanza et G. Laferté définissent l'identification comme «l'action sociale ou l'attribution identitaire extérieure, s'exerçant sur un individu, dans le cadre d'une institution sociale». Ils emploient par ailleurs l'expression image sociale, qui désigne le résultat de la production des discours et des représentations que des élites cherchent à imposer à des ensembles plus larges. Enfin, ils entendent par appartenance les pratiques d'appropriation ou de refus des identifications et des images. "L'appartenance relève de la participation des individus à la chose collective, au groupe» (Avanza, Laferté, 2005, p. 142-144). 
de l'appartenance à un ou plusieurs groupes sociaux ou catégories "॥ (Kroskrity, 1999, p. 111). Néanmoins, E. Versluys regrette le fait que, si un grand nombre d'auteurs qui traitent de la relation entre langage et identité soulignent la nature construite de cette dernière, cela reste souvent au stade de la théorie et n'est pas réellement mis en pratique dans les analyses (Versluys, 2007, p. 93-97). Cette approche constructiviste de l'identité peut être rapprochée de la position assumée par l'analyse du discours dite «à la française », qui considère plus généralement que le discours n'est pas un mode d'accès aux représentations du monde - qui permettrait notamment d'avoir accès aux intentions, aux stratégies, aux idées, aux pensées ou aux opinions -, qu'il n'est pas une représentation de la réalité, mais qu'il est lui-même une réalité (KriegPlanque, 2012, p. 40-41). C'est dans cette tendance de l'analyse du discours que s'inscrit ce dossier, et nous nous intéresserons donc principalement ici à des approches constructivistes de l'identité.

Dans une approche philosophique, Paul Ricœur souligne lui aussi l'importance du langage dans la construction identitaire : cette dernière se fait, selon lui, à travers l'activité narrative. En effet, depuis la psychanalyse, le moi n'est plus conçu comme une conscience unifiée, mais comme une personnalité divisée entre le moi, le surmoi et l'inconscient - P. Ricœur parle de «cogito brisé ». Le récit autobiographique apparaît donc comme le moyen de satisfaire le besoin qu'a tout être humain de donner une certaine unité à son existence, en permettant à l'individu ou au groupe de se présenter à soi-même comme un tout unifié, et ainsi, de construire, ou de reconstruire son identité. C'est ce que P. Ricœur appelle «l'identité narrative » (Ricœur, 1983-1985 et 1990, dans Kaufmann, 2006). Dans la lignée de cet auteur, un certain nombre de travaux, développés davantage par ceux qui s'intéressent à l'identité individuelle et relevant souvent de la psychologie, mettent en avant le lien entre identité et récit de soi. Si, dans des travaux plus anciens, les récits biographiques étaient vus comme des représentations du monde et des identités, les travaux plus récents relevant de l'approche narrative cherchent à déterminer comment les personnes utilisent les récits dans leurs échanges quotidiens pour construire le sens de ce qu'ils sont (Bamberg et al., 2011, p.183-187). Il s'agit en ce sens d'approches de l'identité que l'on peut qualifier de "constructivistes".

Patrick Charaudeau (2009b), s'intéressant lui aussi davantage à l'identité personnelle à travers la question de l'identité du sujet parlant, souligne quant à lui l'importance de la situation de communication : l'identité n'est pas seulement une construction volontaire et consciente du sujet, elle est aussi contrainte par le contexte. En effet, pour lui, l'identité a deux composantes: l'identité (psycho)-sociale et l'identité discursive. La première est attribuée

3. "Identity is defined as the linguistic construction of membership in one or more social groups or catégories» (Kroskrity, 1999, p.111). 
et reconnue par institutionnalisation. L'identité sociale fonde la légitimité du sujet; c'est elle qui lui donne droit à la parole. Pour cette raison, elle doit être reconnue par les autres (ibid., p. 18-20). La deuxième, l'identité discursive, est construite par le sujet parlant et elle repose sur le besoin d'être cru. C'est un enjeu de crédibilité qui est derrière cette identité discursive. Le jeu entre ces deux identités est déterminé par le dispositif de la situation de communication: en effet, cette dernière détermine l'identité sociale et donne au sujet parlant des instructions quant à la façon de se comporter discursivement (p. 21-23). Mais le sujet peut choisir de respecter ou non ces instructions, et ce faisant, de reconstruire, masquer ou déplacer cette identité sociale. De l'évaluation qu'il fait de la marge de manœuvre dont il dispose dans une situation de communication donnée découle une série de stratégies discursives que P. Charaudeau regroupe autour de trois enjeux : (a) les enjeux de légitimation qui reposent sur la nécessité pour le locuteur de convaincre son interlocuteur que sa prise de parole et sa manière de parler correspondent à la position d'autorité conférée par son statut (autrement dit, il s'agit, pour le locuteur, de montrer que son identité discursive correspond à son identité sociale); (b) les enjeux de crédibilité qui reposent sur la nécessité de faire croire à l'interlocuteur que ce qui est dit est « digne de foi »; (c) les enjeux de captation qui conduisent à faire en sorte que l'interlocuteur adhère à ses propos. Ces trois stratégies construisent une identité discursive propre au sujet (p. 26-28).

Par ailleurs, la question de l'identité en amène une autre, soulevée par de nombreux auteurs (par exemple, Charaudeau, 2009a et 2009b; Moirand, 2012) et qui lui est indissociable : celle de l'altérité et de la relation à l'autre. En effet, comme on vient de l'évoquer, l'identité se construit à travers un «processus réciproque [...] de reconnaissance de l'autre et de différenciation vis-à-vis de cet autre » (Charaudeau, 2009b, p.15). L'identité étant, en premier lieu, une prise de conscience de soi, c'est la perception de la différence par rapport à l'autre qui constitue la preuve de sa propre existence et donc de sa propre identité (ibid.). Ainsi, s'interroger sur l'identité d'un point de vue discursif passe notamment par l'analyse du répertoire des marques linguistiques à travers lesquelles on se ressemble, ou au contraire, on se différencie de l'autre (Bamberg et al., 2011). C'est ce rapport d'interdépendance que souligne Émile Benveniste lorsqu'il affirme que «la conscience de soi n'est possible que si elle s'éprouve par contraste. Je n'emploie je qu'en m'adressant à quelqu'un, qui sera dans mon allocution un tu» (1972, p. 260, italiques de l'auteur). Autrement dit, le je et le tu se définissent mutuellement. P. Charaudeau (2009b, p. 16) constate par ailleurs que, de façon paradoxale, l'autre, dans sa différence, est souvent perçu de façon négative, ce jugement négatif étant à l'origine des stéréotypes et des préjugés.

Ce «principe d'altérité»-pour reprendre les mots de P. Charaudeau (2009b, p.15) - est lui-même inextricablement lié à la question de la reconnaissance. En effet, l'identité repose, on vient de le dire, sur un processus réciproque de 
reconnaissance de l'autre (ibid., p.15 et 19). C'est également ce que souligne Pierre Bourdieu, s'interrogeant sur la question de l'identité régionale : pour lui, «exister socialement c'est aussi être perçu, et perçu comme distinct» (1980, p.67) - ce qu'il appelle la «distinction ». Cette distinction passe donc par la reconnaissance de la différence. Ainsi, la région est avant tout, selon lui, une représentation dont l'existence dépend de la reconnaissance qui lui est accordée $e^{4}$ (Bourdieu, 1980, p. 63). Cet aspect est aussi présent dans la notion de "performance », utilisée par Mary Bucholtz et Kira Hall pour rendre compte de la construction discursive de l'identité. Cette notion renvoie à la fois à l'idée de mise en scène - du sens premier du terme performance en anglais («spectacle », "représentation ») - et à la dimension performative du langage. Elle correspond à une monstration sociale consciente et délibérée (deliberate and self-aware social display), à une mise en avant de l'identité à l'attention d'un public. Cette « performance » vise la reconnaissance et est souvent utilisée pour résister ou se rebeller (Bucholtz, Hall, 2004, p.380-381). Cette notion renvoie donc aux différentes formes de revendication identitaire. La reconnaissance de l'identité a, en outre, plus de chances de se produire lorsque les responsables des discours producteurs d'identité bénéficient d'une autorité sociale, d'une légitimité, comme dans le cas du discours scientifique ou de celui de l'État (Bourdieu, 1980; Avanza, Laferté, 2005, p.139). Ces aspects mettent donc en évidence le rôle joué par les discours médiatiques et politiques - qui nous intéressent plus particulièrement dans le cadre de la revue Mots - dans la construction de l'identité, étant donné qu'ils peuvent agir comme moteurs de sa reconnaissance.

La question de la relation à l'autre nous conduit, pour finir, à un aspect pointé par M. Bucholtz et K. Hall (2004), qui s'avère essentiel pour comprendre les revendications identitaires socio-politiques en Amérique latine. Il s'agit des relations de pouvoir que sous-tend la question de l'altérité. Ressembler à l'autre ou s'en distinguer relève souvent de relations asymétriques dans lesquelles les caractéristiques et les appartenances ethniques, religieuses, de classe ou langagières du groupe dominant s'imposent comme la norme sociale. En effet, «il y a dans toute société au moins un groupe dominant, considéré comme prestigieux par les autres groupes, autour duquel s'organisent les différences» (Béjoint, 1980, cité par Bonnet, 2011, p.4). En conséquence, les caractéristiques de ce groupe dominant sont moins perçues comme des traits identitaires. En revanche, tout ce qui est perçu comme différent par rapport à cette norme est généralement discriminé, voire nié. Ce phénomène, désigné par M. Bucholtz et K. Hall comme «marquage» (markedness), rend compte de

4. P. Bourdieu souligne d'ailleurs les paradoxes que suppose parfois cette reconnaissance, par exemple dans le cas de l'Occitanie et de la langue occitane, dont la reconnaissance - en tant qu' «occitan»-implique l'imposition d'une norme unique au détriment des divers parlers occitans (Bourdieu, 1980, p. 66). 
l'évaluation souvent négative des différences qui sont perçues comme des déviations par rapport à la norme et qui supposent une hiérarchie entre groupes sociaux (Bucholtz, Hall, 2004, p. 370-372). Cette notion de marquage est à rapprocher de la «stigmatisation » décrite parP. Bourdieu. Pour lui, ce sont les stigmates découlant de la domination d'un groupe sur un autre (d'une région sur une autre dans le cas étudié) qui engendrent la revendication des stigmates, ceux-ci en devenant ainsi les emblèmes (par exemple : "black is beautiful»); autrement dit, ce sont les stigmates eux-mêmes qui fondent la revendication identitaire. Ainsi, si l'on abolit les stigmates, on détruit les fondements mêmes de l'identité (Bourdieu, 1980, p.69-70).

Dans le cas qui nous concerne, ces concepts de «marquage» et de «stigmatisation » permettent d'appréhender ce qui s'est passé et continue, dans une certaine mesure, de se produire en Amérique latine depuis la «conquête» du continent par les Européens. En effet, un groupe social s'est assez rapidement imposé comme légitime, au détriment des autres groupes sociaux qui ont ainsi été «marqués» comme différents, "stigmatisés». Ce processus et les conséquences qui en résultent font de l’Amérique latine un terrain particulièrement fécond pour qui s'intéresse à la question des identités collectives et des conflits qui en résultent, comme nous chercherons à le montrer dans la section qui suit.

\section{Identités en Amérique latine / Identité latino-américaine?}

La dénomination "Amérique latine» suggère en elle-même des pistes de réflexion quant à la question des identités dans ce continent. Tout d'abord, elle évoque d'emblée une certaine idée d'unité. Ainsi, certains auteurs, revendiquant l'idée d'une essence latino-américaine, ont cherché à définir l'identité spécifique de ce continent. Parmi ces essais de définition, on peut distinguer, d'une part, ceux qui considèrent que cette essence se trouve dans les populations amérindiennes (désignées aussi sous le nom de populations autochtones, indigènes, ou encore indiennes) et, d'autre part, ceux qui, reconnaissant le métissage durant la colonisation, estiment que l'essence latino-américaine se trouve dans le métis. D’autres rejettent ces visions essentialistes et l'idée d'une identité déjà préconstruite qu'il faudrait récupérer. Pour eux, l'Amérique latine est toujours à la recherche de sa propre identité (Larraín, 1994, p. 49-57).

En réalité, il convient de rappeler que la plupart des sociétés latinoaméricaines ne sont pas unifiées et qu'il existe une importante diversité culturelle. Cette dernière est le produit d'une rencontre asymétrique et conflictuelle entre trois cultures (amérindienne, africaine et européenne) dont les effets sont encore visibles aujourd'hui. Asymétrique et conflictuelle, car, comme le fait remarquer Alfredo Gomez-Muller, la dénomination «Amérique latine » suggère 
également que dans ce continent, les rapports de pouvoir ont été historiquement construits sur la base de l'exclusion des référents ethnico-culturels non hispaniques ou non «latins »: ceux des peuples natifs et afro-américains (2012, p. 16). En effet, l'identité qui s'est imposée comme étant la norme correspond à celle de la population blanche, descendante des Européens, catholique et hispanophone ou lusophone. En conséquence, les groupes sociaux portant d'autres caractéristiques ethniques, religieuses ou linguistiques ont été «marqués », non seulement comme étant différents, mais surtout comme étant inférieurs. Ces groupes ont ainsi subi des discriminations qui les ont menés, dans certains cas, à revendiquer une identité en l'affichant (en la «performant»).

S’interrogeant sur l'identité latino-américaine, Jorge Larraín (1994) revient ainsi sur plusieurs moments-clés de l'histoire de l'Amérique latine, au cours desquels la question de l'identité a fait l'objet de questionnements collectifs importants et remarque que ces moments correspondent à des périodes de crise, où les modes de vie traditionnels de certains groupes sont menacés. La première d'entre elles correspond selon lui à celle initiée par la conquête et la colonisation du continent américain par les Européens. En effet, à cette période, la domination, voire parfois l'élimination des populations autochtones s'est accompagnée d'une construction de l'image de l'indio («indien ») comme un « autre » inférieur (Larraín, ibid.). On peut d'ailleurs remarquer que la désignation indio, attribuée à ces populations par les Espagnols à leur arrivée sur le continent, leur permettait à la fois d'introduire une distinction à caractère ethnique entre les deux groupes (colonisateurs et colonisés) et d'homogénéiser des sociétés très diverses tout en les désignant comme un Autre différent d'eux-mêmes (Bonfill Batalla, 1995, dans Heymann, 2013, p.11-12). Par ailleurs, en espagnol, le terme indio entre aujourd'hui encore dans la construction de plusieurs expressions dépréciatives (par exemple hacer el indio : «faire le zouave ») et a lui-même une valeur péjorative dans de nombreux pays d'Amérique latine (Jarak, 2013).

Par la suite, à partir du début du $19^{\mathrm{e}}$ siècle, les guerres d'indépendance visà-vis de la couronne espagnole, puis la construction des États-Nations, ont été menées essentiellement par les élites criollas ${ }^{5}$, fortement influencées par l'esprit des Lumières, le libéralisme britannique, puis le positivisme et la pensée rationaliste. Ces élites dirigeantes ont cherché à construire des Nations «modernes », inspirées par le modèle européen, ce qui les a conduites à nier la diversité linguistique, ethnique et culturelle de leurs sociétés (Gomez-Muller, 2012, p. 13-15). En s’appuyant sur des théories pseudo-scientifiques dévelop-

5. Le terme criollo désigne les descendants des colonisateurs européens nés sur le sol américain. C'est d'ailleurs de ce mot espagnol qu'est venu le français créole (autrefois criole) : «personne de pure race blanche née aux colonies », selon une citation rapportée par le TLFI. Le terme espagnol a lui-même été emprunté au portugais crioulo : «Noir né dans les colonies» (TLFI, article créole). 
pées au $19^{\mathrm{e}}$ siècle (par exemple, les théories raciales d'inspiration darwiniste de Juan Alberdi (1852) ou celles de Domingo F. Sarmiento - la fameuse lutte de la «civilisation» contre la «barbarie»), les élites criollas pouvaient plus facilement justifier le racisme et la discrimination envers des groupes ethniques, majoritaires en nombre dans certains cas, mais minoritaires sur le plan politique : les peuples autochtones et les populations noires, qui faisaient déjà l'objet de discriminations depuis la colonisation (Larraín, 1994, p. 38-47). En d'autres termes, on a affaire à la construction d'une identité nationale par le haut, correspondant à une volonté d'uniformiser la population en faisant disparaître la diversité ethnique, ou du moins, en en intégrant seulement certains traits culturels considérés comme meilleurs (Grimard, 2008; Helg, 2004; Montes, 2015; Larraín, 1994).

Pour illustrer cet état de fait, on peut mentionner les récits historiques portant sur cette époque et qui mettent en valeur la participation des hommes blancs dans la construction des États-Nations. Dans ces récits, la participation des femmes, des Amérindiens et des Noirs est en revanche largement minimisée voire occultée, comme le démontre l'analyse de l'historiographie colombienne réalisée par Angélica Montes (2015) et l'analyse de manuels scolaires à destination des adolescents colombiens (Serrano, 2009). Néanmoins, si, dans des pays comme l'Argentine, qui ont subi un processus de «blanchiment» de la population, encouragé et financé par les élites criollas aux $19^{e}$ et $20^{\mathrm{e}}$ siècles, la construction de l'identité nationale semble davantage reposer sur une vision unique et homogène qui nie la diversité culturelle, dans des pays où la composante amérindienne constitue une part importante de la population, comme le Pérou ou le Mexique, l'identité nationale est moins associée à l'idée d'une population uniformément blanche (Larraín, 1994, p. 60-64). On voit ainsi l'apparition, dans la seconde moitié du $19^{\mathrm{e}}$ siècle, des premiers travaux dits indigénistes; néanmoins, si ces derniers reconnaissaient l'exploitation séculaire des populations amérindiennes, ils prônaient le métissage comme solution au «problème indien» (Heymann, 2013, p. 60-62).

Il faudra donc attendre la première moitié du $20^{\mathrm{e}}$ siècle pour voir le début des mobilisations sociales et un renouvellement de la pensée indigéniste, appelant désormais à modifier les stéréotypes négatifs sur les Amérindiens et prônant des réformes sociales en leur faveur. Cependant, la modernisation qui s'est imposée au cours de la première partie du $20^{\circ}$ siècle et l'impérialisme des États-Unis ont contribué à renforcer l'exclusion de certains secteurs de la société, notamment des "minorités» ethniques. Ce phénomène s'accentue encore dans la seconde moitié du $20^{\mathrm{e}}$ siècle et mènera à une radicalisation des classes populaires, avec des revendications d'ordre politique et social. Cette dernière entraînera, dans les années soixante-dix, une vague de coups d'État suivis de dictatures militaires dans plusieurs pays latino-américains (Larraín, 1994, p. 38-49). Ces événements vont susciter de nouvelles réflexions autour 
de la question de l'identité latino-américaine et une remise en question du modèle européen comme solution aux problèmes du continent, se manifestant principalement à travers les tendances mentionnées plus haut - soit il existe une identité latino-américaine originale qu'il faudrait retrouver, soit cette dernière reste à construire (Larraín, 1994, p. 49-57). Dans les deux cas, les discours identitaires sont liés à des causes politiques, sociales et économiques et entraînent notamment d'importants enjeux en termes de participation politique et de gestion des ressources naturelles économiquement essentielles pour les classes socio-économiques les plus défavorisées.

Ainsi vont émerger, au cours du $20^{\mathrm{e}}$ siècle, un certain nombre de mouvements ou d'initiatives menés en réaction à la stigmatisation historique de certains groupes ethniques ou sociaux. La Colombie par exemple, à la suite de l'Assemblée constituante de 1991, a reconnu le caractère multiculturel et pluriethnique de sa population en promulguant la loi 70 de 1993, qui accorde aux communautés noires le droit à la propriété de certaines terres et offre de nouveaux espaces de représentation et de participation politique (Montes, 2015). On peut citer aussi les mouvements qui revendiquent les droits des populations dites indigènes dans différents pays, comme le mouvement des (néo-) zapatistes au Mexique ou des Mapuches au Chili. Par ailleurs, on voit dès la deuxième moitié du siècle dernier, la mise en place d'initiatives interétatiques qui illustrent la volonté de construire une certaine unité latino-américaine en opposition à la domination économique, voire politique, des États-Unis, et ne sont pas sans rappeler le rêve d'union continentale de Simón Bolívar. C'est le cas par exemple du MERCOSUR ${ }^{6}$ ou de la Communauté andine des nations ${ }^{7}$ qui cherchent à regrouper les pays latino-américains autour d'orientations économiques communes. Plus récemment, l'arrivée au pouvoir de gouvernements dirigés par des leaders issus de groupes ethniques discriminés s'est accompagnée d'autres types d'initiatives supranationales comme la création de la $\mathrm{CELAC}^{8}$ (dont sont volontairement exclus les États-Unis, le Canada et les territoires d'outremer des pays européens), ou de l'ALBA ${ }^{9}$ (impulsée par Hugo Chávez et proposée comme une alternative à l'ALCA - Aire de libre commerce des Amériques - dont les États-Unis sont le principal moteur), avec des revendications plus politiques et identitaires. Ces gouvernements progressistes, comme celui d'Evo Morales en Bolivie, d’Hugo Chávez et de son successeur

6. Marché commun du Sud, créé en 1985 par le Brésil, l'Argentine, le Paraguay et l'Uruguay pour instaurer un espace de libre circulation des biens. Le Venezuela a rejoint ce marché commun en 2012 et d'autres pays comme la Colombie, le Pérou ou l’Équateur sont des pays associés.

7. Organisation d'intégration subrégionale regroupant aujourd'hui la Bolivie, la Colombie, l’Équateur et le Pérou.

8. Communauté d'États latino-américains et caribéens : créée en 2010 comme une alternative à l’Organisation des États américains (OEA), elle regroupe 33 États de l'Amérique centrale, du Sud et des Caraïbes.

9. Alliance bolivarienne pour les peuples de notre Amérique : organisation promouvant l'intégration - économique principalement - des pays d’Amérique latine et de la Caraïbe. 
Nicolás Maduro au Venezuela, de Rafael Correa en Équateur ou de Daniel Ortega au Nicaragua, reconnaissent et revendiquent le caractère métis de la population en promouvant certaines mesures en faveur des populations auparavant exclues, ainsi que l'unité latino-américaine. À titre d'exemple, Daniel Ortega a pris l'initiative de ne plus commémorer, à partir du 12 octobre 2008, "el día de la raza» (la journée de la race), mais « el día de la resistencia indígena» (la journée de la résistance indigène). Ces différentes revendications et initiatives sont menées dans un but d'opposition à la domination politique, culturelle et économique des élites locales, mais aussi des États-Unis, et dans une moindre mesure de l'Europe. Dans ce cadre, l'idée d'une certaine identité latino-américaine est revendiquée comme un élément homogénéisateur face à la mondialisation (Poccioni, 2001, p. 394). Enfin, on peut citer les législations mises en place dans certains pays, comme l'Uruguay, l'Argentine ou la Colombie, au sujet des violences faites aux femmes et du mariage homosexuel, en réponse aux revendications d'une autre catégorie de population stigmatisée, les femmes et les communautés LGBT (Lesbiennes, gays, bisexuels et transgenres), problématiques qui, si elles ne sont pas spécifiques de l'Amérique latine, n'en sont pas moins importantes dans ce continent.

On peut donc constater que la question des identités sociales, politiques, linguistiques ou de genre, liée à l'existence de groupes sociaux minorisés à l'intérieur des pays latino-américains, résulte notamment du processus historique de colonisation, des indépendances et de la construction des États-Nations. Ainsi, si nous appréhendons l'identité comme faite - construite - à travers les pratiques discursives (Bamberg et al., 2011, p.178), cela ne signifie pas pour autant que cette dernière n'ait pas de fondement. Autrement dit, la domination et la stigmatisation de groupes sociaux sont des faits bel et bien avérés, mais nous considérons que l'identité - entendue ici comme le processus par lequel un individu ou une collectivité prend conscience de son existence et se positionne par rapport à d'autres groupes sociaux - est un construit, dans lequel le discours joue un rôle fondamental, comme le montrent les articles de ce dossier.

\section{Notre dossier « Identités et conflits »}

Les différentes contributions qui constituent ce dossier s'attachent donc, à travers diverses études de cas, à analyser comment ces identités collectives se construisent, se disputent et se négocient à travers les discours politiques et médiatiques, ou encore à travers ceux de locuteurs «ordinaires» réagissant face à ces questions.

Lætitia Rouvière analyse ainsi la construction d'une identité transfrontalière aymara entre le Chili, le Pérou et la Bolivie dans le discours des dirigeants locaux et de leurs financeurs, les institutions internationales. L'auteure montre 
comment une identité historiquement construite de façon négative - celle des populations autochtones aymaras - peut être, en quelque sorte, mise en avant pour être renversée, dans un processus d' «inversion du stigmate» (Goffman, 1975, cité par Rouvière dans ce numéro). En d'autres termes, l'identité «marquée» n'est plus cachée, mais montrée. Dans le cas traité par L. Rouvière, cette mise en scène de l'indianité représente en fait un moyen pour satisfaire les conditions de bailleurs de fonds, qui cherchent à promouvoir le « développement avec identité». C'est pour cette raison que l'auteure parle d'une «ethnicisation » de l'identité aymara. L'auteure montre ainsi comment, dans ce cas particulier, «la revendication d'identités autochtones en Amérique latine ne renvoie [...] pas nécessairement aux mobilisations progressistes les plus visibles », mais peut, au contraire, répondre à une logique néo-libérale.

Toujours à propos des identités ethniques, Karla Avilés décrit au contraire un cas d'assimilation au sens où l'entend P. Bourdieu, c'est-à-dire un processus visant à faire disparaître les signes du stigmate afin de produire une image de soi conforme à l'identité légitime (1980, p.69). Cette auteure traite en effet du cas de la langue nahuatl, devenue un stigmate que portent les communautés de Santa Catarina (Mexique) étudiées dans son article. Dans ce cas, la communauté nahua ${ }^{10}$ est «marquée » et de ce fait subit une domination linguistique qui imprime ses marques dans le discours des locuteurs du nahuatl, où elle provoque, bien souvent, le silence ou le non-dit. L'un des apports de cet article réside dans le constat tiré des conversations informelles entre des représentants de cette communauté et l'auteure, qui observe que sa présence en tant que chercheure dans ces conversations facilite une «catharsis identitaire », c'est-àdire qu'elle favorise l'expression du non-dit dans une communauté qui autocensure la pratique de sa propre langue et ne l'enseigne presque plus aux enfants.

Auprès de la question des identités ethniques, c'est d'identité nationale qu'il s'agit avec la contribution de Morgan Donot. S'inscrivant dans une approche argumentative, l'auteure analyse la manière dont deux présidents argentins, Carlos Menem et Néstor Kirchner, construisent, chacun à leur manière, une identité nationale argentine autour de la «cause des Malouines". On sait qu'en 1982, les îles Malouines furent à l'origine d'une guerre entre l'Angleterre et l'Argentine, perdue par celle-ci. Alors que Menem, dans le but de favoriser une réconciliation du pays, prône la « démalouinisation », montrant ainsi une volonté de faire oublier cette guerre - et surtout le fait que c'est la dictature militaire qui en fut le responsable direct -, Kirchner prône la «rema-

10. Nahua est le terme générique qui s’applique à l'ensemble du groupe linguistique concerné, et s'oppose aux termes qui désignent les variantes à l'intérieur de ce groupe; nahuatl est le vocable désignant les variantes linguistiques parlées surtout au centre du Mexique, y compris dans la région concernée par cet article (pour d'autres variantes, où le phonème $t$ est absent ou rare, par exemple la Huastèque de l'État de Veracruz, on parle de nahuat). Enfin, le composé nahuatophone désigne les locuteurs du groupe linguistique nahua. 
louinisation » qui consiste à imposer un devoir de mémoire et dans le même temps, à désigner un ennemi interne, la hiérarchie militaire de l'époque. Ainsi, les responsabilités ne sont plus masquées derrière une certaine « unité nationale », comme le prétendait la « démalouinisation» promue par Menem.

De son côté, Clémentine Berjaud s'intéresse à l'identité politique et sociale des récepteurs des discours d'Hugo Chávez au Venezuela. S'appuyant sur l'étude des mots gauche, droite et socialisme, elle explore le sens de ces catégories politiques sur la base d'entretiens avec des citoyens ordinaires, afin de déterminer si ces dernières sont porteuses des conflits sociopolitiques présents dans ce pays. L'auteure constate que le sens donné à ces mots dépend de l'appartenance sociale et politique des locuteurs : l'interprétation de gauche ou de droite n'est pas la même pour un partisan ou pour un opposant d'Hugo Chávez. Autrement dit, ces mots servent davantage à définir des camps politiques, auxquels les locuteurs s'identifient ou dont ils se distancient, qu'à désigner les concepts auxquels ils renvoient. Il en ressort donc un usage essentiellement polémique de ce vocabulaire, et un flottement référentiel.

Enfin, ce dossier accorde également une place aux identités de genre, problématique qui, comme on l'a souligné, n'est pas exclusive à l'Amérique latine. Dans son article, Myriam Hernández s'intéresse aux campagnes de prévention menées par le premier gouvernement de Michèle Bachelet, au Chili, contre les violences faites aux femmes au sein du couple. Analysant la construction de l'identité respective des hommes et des femmes acteurs de ces violences, l'auteure montre l'évolution, dans le discours étatique, de la représentation des rapports de pouvoir à l'intérieur du couple : on passe d'une représentation de la femme mère, épouse et toujours victime à la représentation d'une femme empoderada (consciente de son propre pouvoir) - qui peut faire penser à l'image de la présidente elle-même. Au fil de ces campagnes se construit peu à peu un nouveau modèle de famille, dont les membres tendent vers l'égalité.

Les différentes réflexions menées dans ce dossier explorent donc les liens entre identité et langage : elles mettent par exemple en évidence des cas d'assimilation de l'identité dominante, ou au contraire, d' «inversion du stigmate »; plus largement, elles soulignent le rôle que joue le discours dans la construction de l'identité ethnique, linguistique, nationale, sociopolitique, ou encore dans la construction des rapports de genre. Elles montrent ainsi que la question des identités collectives en Amérique latine est une question éminemment politique.

\section{Références}

AlBERDI Juan Bautista, 1852, Bases y puntos de partida para la organización política de la República Argentina, derivadas de la ley que preside el desarrollo de la civilización en América del Sur, Valparaíso, Imprenta del Mercurio. 
Avanza Martina, LAferté Gilles, 2005, "Dépasser la "construction des identités"? Identification, image sociale, appartenance », Genèses, nº 61 (4), p. 134-152.

Bamberg Michael, De Fina Anna, Schiffrin Deborah, 2011, "Discourse and identity construction ", Handbook of Identity Theory and Research, S. J. Schwartz, K. Luyckx, V. L. Vignoles éd., New York, Springer-Verlag New York Inc., vol. I, p.177-199.

Benveniste Émile, 1972 [1958], «De la subjectivité dans le langage», Problèmes de linguistique générale, chap.XXI, Paris, Gallimard.

BONNET Valérie, 2011, «"Don’t call me Nigger, Whitey”. L'autodésignation de la communauté afro-américaine et la construction identitaire», Communication, $\mathrm{n}^{\circ} 28$ (2), p.1-28.

BonfILl BatAlLA Guillermo, 1995, «El concepto de indio en América. Una categoría de la situación colonial», Obras escogidas, vol. I, México, INI-INAH.

BouRdieu Pierre, 1980, "L'identité et la représentation. Éléments pour une réflexion critique sur l'idée de région », Actes de la recherche en sciences sociales, $\mathrm{n}^{\circ} 35$, L'identité, p. 63-72.

BRUBAKER Rogers, 2001, "Au-delà de l'“identité” ", Actes de la recherche en sciences sociales, vol.IV, nº139, p.66-85, http://doi.org/10.3917/arss.139.0066 (consulté le 2 septembre 2015$)$.

Bucholtz Mary, Hall Kira, 2004, "Language and identity », A Companion to Linguistic Anthropology, A. Duranti éd., Oxford, Blackwell, p. 369-394.

CHARAUDEAU Patrick, 2009a, «Identité linguistique, identité culturelle : une relation paradoxale», http://www.patrick-charaudeau.com/Identite-linguistique-identite.html.

- 2009b, «Identité sociale et identité discursive. Un jeu de miroir fondateur de l'activité langagière», Identités sociales et discursives du sujet parlant, P. Charaudeau éd., Paris, L'Harmattan, p.15-28.

DORTIER Jean-François, 2004, Le dictionnaire des sciences humaines, Auxerre, Sciences humaines.

Gomez-Muller Alfredo éd., 2012, Constructions de l'imaginaire national en Amérique latine, Tours, Presses universitaires François Rabelais.

GRIMARD Léon, 2008, «Amérique latine : intégration et identité. Problématiques transnationales et supranationales », Département d'anthropologie, Université de Montréal.

Helg Aline, 2004, Liberty \& Equality in Caribbean Colombia, 1770-1835, Chapel Hill, University of North Carolina Press.

Heymann Catherine, 2013, Les Indiens face à la construction de l'État-Nation. Mexique-Argentine 1810-1917, Paris, CNED.

JARAK Diego, 2013, «Les Indiens face à la construction de l'État-Nation en Argentine», communication au séminaire du LIRA, Mémoires, Identités, Territoires, Université Rennes 2.

KaUfmann Jean-Claude, 2006, "Identité», Dictionnaire des sciences humaines, S. Mesure, P. Savidan éd., Paris, PUF, p. 593-595.

KrIeg-Planque Alice, 2012, Analyser les discours institutionnels, Paris, Armand Colin. KROSKRITY Paul V., 1999, «Identify », Journal of Linguistic Anthropology, ${ }^{\circ}$ 9 (1-2), p. 111114, http://doi.org/10.1525/jlin.1999.9.1-2.111 (consulté le 2 septembre 2015). 
LARRAÍn Jorge, 1994, "La identidad latinoamericana. Teoría e historia », Estudios Públicos, $\mathrm{n}^{\circ} 55, \mathrm{p} .31-64$.

MoIRAnd Sophie, 2012, "Préface», Chikungunya : la médiatisation d'une crise. Presse, humour, communication publique, B. Idelson, G. Legeden éd., EME, p.11-21.

MONTES Angélica, 2015, La représentation du sujet noir dans l'historiographie colombienne. Le cas de Carthagène des Indes (1811-1815), Paris, L'Harmattan.

Poccionı María Teresa, 2001, "Identidad y discurso", Cuadernos de la Facultad de Humanidades y Ciencias Sociales, $\mathrm{n}^{\circ}$ 17, p. 389-394.

Ricceur Paul, 1983-1985, Temps et récit, 3 vol., Paris, Le Seuil.

- 1990, Soi-même comme un autre, Paris, Le Seuil.

Sarmiento Domingo Faustino, 2011 [1851], Facundo. Civilización y barbarie. Vida de Juan Facundo Quiroga, Buenos Aires, Altamira.

SERRANo Yeny, 2009, "Image des populations dites "minoritaires" (Noirs, Amérindiens, Métis, femmes) véhiculée par les manuels scolaires aux adolescents/es en Colombie », Amériques Noires. Réflexions, hors-série, p. 63-75.

VERSLUYS Eline, 2007, "The notion of identity in discourse analysis. Some "discourse analytical” remarks", RASK. Internationalt Tidsskrift for Sprog Og Kommunikation, $\mathrm{n}^{\circ} 26$, p. 89-99. 